\title{
GENERALISATION OF SUBMARINE FEATURES ON NAUTICAL CHARTS
}

\author{
Eric Guilbert, Xunruo Zhang \\ Department of Land Surveying and Geo-Informatics \\ The Hong Kong Polytechnic University \\ Hung Hom, Kowloon, Hong Kong \\ 1seguil@polyu.edu.hk, crystal_zxr@hotmail.com
}

Invited Paper, Commission II, WG II/2

KEY WORDS: Model generalization, GIS, Landscape representation, Cartography, DEM/DTM representation

\begin{abstract}
:
On most large scale and middle scale maps, relief is represented by contours and spot heights. In order to adapt the representation to the scale, the terrain is generalised either by smoothing or filtering the terrain model or by simplifying the contours. However this approach is not applicable to nautical chart construction where terrain features are selected according to their importance for navigation. This paper presents an approach for the consideration of feature attributes in the generalisation of a set of contours with respect to nautical chart constraints. Features are defined by sets of contours and a set of generalisation operators applied to features is presented. The definitions are introduced in a multi-agent system in order to perform automatic generalisation of a contour set. Results are discussed on a case study and directions for future work are presented.
\end{abstract}

\section{INTRODUCTION}

Nautical charts provide a schematic representation of the submarine relief and are used by navigators to plan their route. The seafloor is portrayed by isobaths and soundings. Bathymetric generalisation on a nautical chart is driven by two main rules in order to obtain a representation that fits navigation requirements. First, the depth reported on the chart can never be deeper than the real depth and navigation hazards such as reefs must be emphasised in order to ensure safety of navigation. Second, navigators do not see the seafloor and rely on the chart to plan their routes. Therefore, relevant fairways or berths must be highlighted.

In comparison with topographic maps, nautical charts provide a more schematic representation of landforms. Starting from the seafloor modelled by a set of soundings and isobaths extracted from the bathymetric database, the cartographer would work in practice by selecting spot soundings and isobaths according to the relevance of the submarine features they model. Sounding position and depth cannot be modified so that spot soundings are selected from the database. Isobaths displayed on the chart can be modified to emphasise characteristic features (which in this domain are mostly prominences and depressions). An isobath modelling a reef is kept and may be enlarged while an isobath marking a depression may simply be omitted if it does not bring relevant information for navigation. On one hand, the chart contains highly accurate information provided by spot soundings while on the other hand great prominence is given to lesser depths. As reported in (NOAA, 1997, p. 4-11), "[cartographers] do, deliberately and knowingly, and on behalf of the navigator, include all lesser depths within a contour even if it means that [their] catch includes many deep ones as well".

As a consequence, contour generalisation obtained by smoothing the terrain and regenerating the contours as done for topographic maps does not yield appropriate results. While terrain generalisation on topographic maps is more relevant to cartographic generalisation where the objective is to provide a legible representation of the terrain, bathymetric generalisation on nautical charts pertains to model generalisation where submarine features are selected according to their meaning for the purpose of the map.
In order to consider submarine features in the process, they must be identified from the contours and recorded in a topological data structure. Features are defined by sets of contours as perceived by the navigator. Constraints and operators can be defined and applied to contours based on feature data. The present paper discusses the constraints and operations to consider for featuredriven generalisation of a contour map. While most methods for contours are relevant to cartographic generalisation, the approach is more relevant to model generalisation as operators apply to features according to their meaning.

This paper defines continuous operators based on the snake model that take into account feature-related constraints and discrete operations that modify the feature data structure in the context of nautical chart generalisation. Operations that apply only to single contours such as smoothing and conflicts occurring between contours within a feature are not addressed.

The paper first reviews existing work on contour generalisation and terrain feature representation on contour maps. New generalisation operators are defined in section 3. Both constraint definitions and the updating of the data structure are addressed. In section 4, operators are introduced into a multi-agent system and applied to nautical chart generalisation. Results are presented and discussed. Finally, conclusions and perspectives for future work are presented.

\section{PREVIOUS WORKS}

\subsection{Contour generalisation}

Automatic generalisation of contour lines has been studied for long and several approaches have been developed for the simplification of contour shape and the removal of legibility conflicts. Different criteria are used such as the distance between the lines (Gökgöz, 2005; Li and Sui, 2000), the slope gradient (Mackaness and Steven, 2006), the medial axis transform (Matuk et al., 2006) or the drainage system (Ai, 2004). These methods refer to cartographic generalisation where the objective is to improve the legibility and aesthetic of the map by selecting or simplifying the 


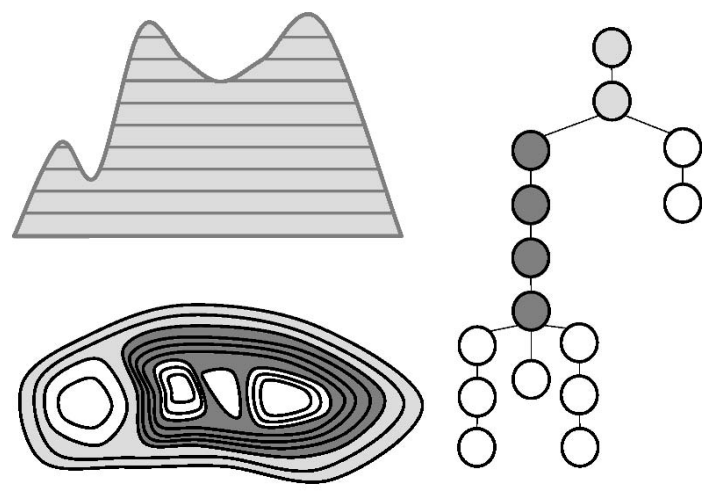

Figure 1: Contour tree and map. Branches at the bottom of the structure (white contours) represent peaks and pits.

contours. The main advantage of these methods on simple line smoothing operations is that local terrain characteristics can be taken into account to maintain the shape of the contours and the topological relationships between the contours. But the character of features modelled by the contours is not considered and only simplification is performed.

Other line generalisation operators can also apply to contours such as aggregation, smoothing, displacement or exaggeration. Earliest approaches consisted in defining dedicated rules for specific operations or applications such as road or coastline simplification (Nickerson, 1988; Mustière, 1998; Wang and Müller, 1998). However, a difficulty for process automation is that applying these methods is based on rules which apply for single lines but which are limited for processing groups of lines as they cannot handle side effects and the result may depends of the order in which operations are performed. Therefore, more recent techniques are based on the minimisation of constraints expressing conditions to satisfy via continuous optimisation techniques (Harrie and Weibel, 2007).

Optimisation techniques are mostly based on energy minimisation approaches such as snakes (Burghardt and Meier, 1997) or elastic beams (Bader, 2001). Methods apply to roads (Burghardt and Meier, 1997), buildings (Bader et al., 2005) or contour lines (Guilbert and Saux, 2008). The last method, based on snakes, takes into account position constraints so that a line can be smoothed while preserving a minimal distance with neighbouring objects or by pushing the line in one direction. It applies to isobathymetric line generalisation but terrain feature information is not considered and interaction with the cartographer is required.

\subsection{Feature representation on a contour map}

The most common topological data structure applied to a contour map is the contour tree built on inclusion relationships between the contours (Cronin, 1995). Two kinds of features are extracted: peaks (or more generally, prominences) and pits (or more generally, depressions), which correspond to branches containing leaves of the contour trees (Kweon and Kanade, 1994) (Figure 1). However this representation does not take into account the different levels at which terrain features can be described. Only features at the highest level of detail are defined and depending on the level of detail considered, it is not possible to know which type of feature a given contour belongs to.

Characterisation of features at different levels from contours on a terrain model was tackled by Chaudhry and Mackaness (2008). Prominences are defined first by morphometric classification of
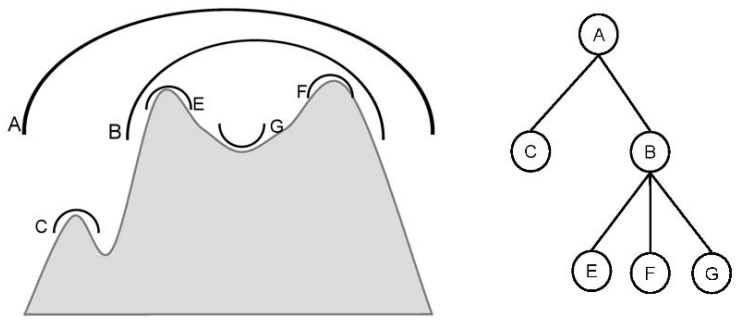

Figure 2: Terrain features and feature tree identified from Figure 1 contour tree (Zhang and Guilbert, 2011).

the DTM. Prominence extent is then defined by the largest contour containing the summit and no other higher point. A data structure storing terrain features at multiple levels which does not require the DTM is proposed by Zhang and Guilbert (2011). Features are defined by groups of contours forming a feature tree (Figure 2). A feature is characterised by a boundary contour representing its spatial extent and all the contours within. A feature is classified as a peak if its boundary contour is lower than all inner contours or as a pit if it is higher than the inner contours. Boundary contours are located at each base of a branch of the contour tree or inside a branch if a change of slope occurs. In comparison with (Chaudhry and Mackaness, 2008), more features are defined as one summit can be contained by several features at different levels.

\section{FEATURE GENERALISATION}

\subsection{Generalisation constraints and operations}

Generalisation requirements can be organised according to different classes of constraints (Beard, 1991). Referring to Ruas and Plazanet (1997)'s classification, constraints in isobath generalisation for nautical charts can be categorised into:

- The legibility constraint: generalised contours must be legible by observing a minimum size or distance between them;

- The position and shape constraints: absolute position and shape of contours must be maintained. Relative distance between contours must be preserved as much as possible;

- The structural and topological constraints: spatial relationships between contours are maintained. Distribution and mean distance between contours are preserved;

- The functional constraint, specific to the purpose of the map: on a nautical chart, functional constraints are the safety constraint indicating that a reported depth cannot be greater than the real depth and the preservation of navigation routes.

The first three constraints (legibility, position and shape) apply on individual contours or locally on groups of contours. Conflicts can be detected and appropriate methods mentioned in section 2.1 can be applied. Structural and topological constraints consider groups of contours. The objective is to maintain morphological details by preserving groups of contours corresponding to terrain features. Constraints are not expressed only at the local level but also at more global levels on larger features. Topological relationships between contours can be recorded in a contour tree however more global information about the terrain morphology at different levels is described in the feature tree (Zhang and 
Guilbert, 2011) which records topological relationships between features modelled by contours.

Functional constraints apply to both single contours or groups of contours depending on the purpose. The terrain representation obtained on the chart is more schematised than on a topographic map as the objective is to clearly mark features on the seafloor rather than to provide a detailed description of the seafloor. Functional and structural constraints can be spatial and semantic as they include constraints related to the type of features and their relationships. On a nautical chart, generalising isobathymetric lines obeys to strict functional constraints:

- An isobath can only be moved towards greater depths;

- An isobath can only be removed if the new representation is not deeper than the original representation;

- Adjacent isobaths can be merged if they are at the same depth and the area in between is deeper;

- Isobaths delineating fairways must be preserved;

- Isobaths characterising features not relevant for navigation can be removed.

Integrating functional constraints in a generalisation process requires the classification of features as whether a too small isobath must be enlarged or removed depends on the type of feature it belongs to. Therefore, functional constraints can be expressed as semantic and spatial constraints on features.

- A feature on the chart must be large enough to contain a sounding marking its deepest or shallowest point.

- A pit cannot be enlarged or aggregated;

- A too small or not relevant pit is removed;

- A peak cannot be removed;

- A too small peak is enlarged or aggregated with an adjacent peak;

- Position of boundary lines of fairways (corresponding to elongated pits at global levels in the feature tree) must be maintained.

As a consequence, generalisation operations apply to features according to the type of conflict and constraints. Generalisation operators applying to features are:

- Selective omission: the feature is deleted with all its contours. Inner features are also deleted. Neighbouring or parent features in the feature tree may be updated;

- Enlargement: contour lines defining the feature boundary are enlarged. The feature tree is not modified;

- Aggregation: adjacent peaks are aggregated into one larger peak by merging their boundary contours. Boundary contours must be at the same elevation. The feature tree and composition of neighbouring features can be modified;
Operations are of two types: continuous operations which do not modify the feature tree or the feature definition and discrete operations which trigger modification of the data structure by creating or removing features or modifying feature attributes. Aggregation is performed in two steps: a continuous deformation filling the gap between boundary segments and a discrete operation joining the feature boundaries.

The following section defines a model for performing continuous operations. Constraints are presented for enlargement and aggregation. On top of enlargement and aggregation performed on peaks, reduction and split may also be performed on pits. However, these operators are hardly used as, in model generalisation, if a feature is considered not relevant, it is simply removed. Nonetheless, these operations can be easily added in the model as their definitions are similar to aggregation and enlargement.

\subsection{Continuous operations}

3.2.1 The snake model Snakes were originally derived by Kass et al. (1987). They are lines defined with their own energy from their geometrical features. A snake is defined by a parametric curve $f(s), s$ being the curvilinear abscissa, which is at an equilibrium position when its global energy is minimal. The global energy of the snake is expressed as

$$
E_{\text {snake }}(f)=\int_{a}^{b}\left[E_{\text {int }}(s)+E_{\text {ext }}(s)\right] d s
$$

where $E_{\text {int }}$ is the internal energy and $E_{\text {ext }}$ is the external energy. The internal energy controls the shape of the snake and is defined from its derivatives:

$$
E_{\text {int }}(s)=\frac{1}{2}\left(\alpha(s)\left|f^{\prime}(s)\right|^{2}+\beta(s)\left|f^{\prime \prime}(s)\right|^{2}\right)
$$

where $\alpha$ and $\beta$ are shape parameters, usually set by the user. The external energy represents application constraints and is not related to the shape of the curve. Performing a generalisation operation consists in applying the appropriate external energy expressing the conflict to correct. One interest of continuous optimisation method is that different energies corresponding to different constraints can be added up resulting in combining different operators together. The following sections describe the external energies for the continuous operations presented in section 3.1.

3.2.2 Enlargement Enlargement is performed by adding to each point an external energy related to the distance to a sounding $\Omega$ inside the feature: the closer a point to the sounding, the larger the energy. Deformation is performed until points of the boundary are at a distance equal to or larger than a threshold distance $\varepsilon_{\text {enl }}$ from the sounding.

$$
E_{\text {enl }}(s)=\left\{\begin{array}{cl}
\varepsilon_{\text {enl }}^{2}-\|f(s)-\Omega\|^{2} & \text { if }\|f(s)-\Omega\|<\varepsilon_{\text {enl }} \\
0 & \text { otherwise }
\end{array}\right.
$$

3.2.3 Aggregation Aggregation is performed by adding an attractive energy term on boundary segments to merge so that the two features move closer and eventually overlap (Figure 3 ). A discrete operator is then applied to create the feature and update the feature tree (section 3.3.2). The purpose of using this attractive energy is to obtain a smooth deformation and prevent potential conflicts with other neighbouring objects. For two given features $F$ and $G$ whose boundaries are respectively noted $f$ and $g$, the external energy applied to each point of $f$ is given by equation 4. 


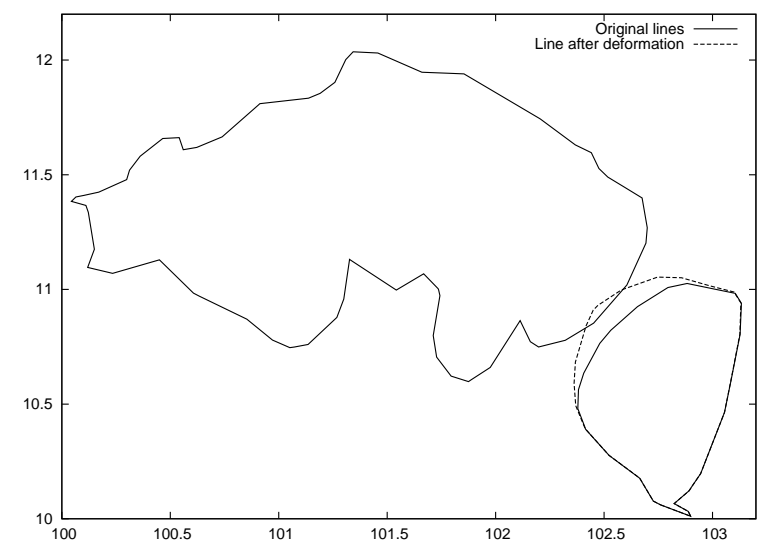

Figure 3: Feature deformation for aggregation.

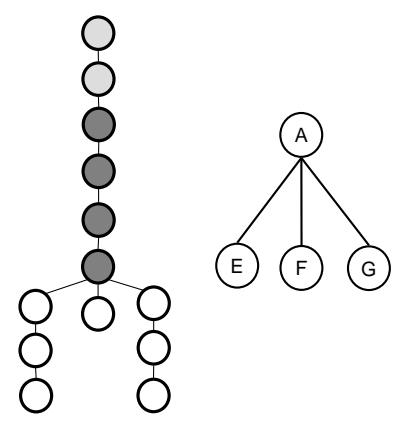

Figure 4: Contour tree and feature tree of Figures 1 and 2 after removal of feature $\mathrm{C}$. Feature $\mathrm{B}$ has been removed because it is of the same class as feature A.

$$
E_{\mathrm{agr}}(s)=\left\{\begin{array}{cl}
d(f(s), g)^{2} & \text { if } d(f(s), g)<\varepsilon_{\mathrm{agr}} \\
0 & \text { if } d(f(s), g) \geq \varepsilon_{\mathrm{agr}} \\
0 & \text { if } f(s) \text { is within } G
\end{array}\right.
$$

with function $d$ the shortest distance from point $f(s)$ to $g$. Deformation is applied to points of $f$ which are at a distance smaller than threshold $\varepsilon_{\text {agr }}$. Deformation is stopped when $f$ and $g$ intersect. The process also requires that the angle formed by intersecting segments is large enough to get a smooth junction of both lines.

3.2.4 Distance constraint In order to prevent creation of new conflicts during the process, a distance constraint is also added to keep features away from their neighbours obtained from the feature tree. If the distance between the line $f$ and those features is smaller than the legibility distance $\varepsilon_{\text {leg }}$, an external energy is added to push back the line into its admissible area. The energy is defined by:

$$
E_{\mathrm{enl}}(s)=\left\{\begin{array}{cl}
\varepsilon_{\mathrm{leg}}^{2}-d(f(s), g)^{2} & \text { if } d(f(s), g)<\varepsilon_{\mathrm{leg}} \\
0 & \text { otherwise }
\end{array}\right.
$$

where $d(f(s), g)$ is the distance between $f(s)$ and a neighbouring feature $g$.

\subsection{Discrete operations on features}

3.3.1 Selective omission Removing a feature from the feature tree implies removing all its contours from the contour tree. If the parent feature is left with one descendant feature of the same class, the descendant feature is removed from the feature tree as it characterises the same feature with a smaller extent (Figure 4).
3.3.2 Feature aggregation The aggregation operation presented in the previous section results in two overlapping features. A new isobath is obtained by aggregating the two intersecting boundaries. Aggregation can lead to the removal or creation of features and the new isobath can belong to a new feature or to an existing one (Figure 5).

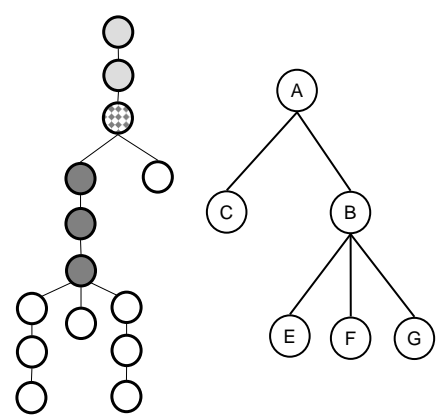

Figure 5: Aggregation of features B and C. The merged contour (hashed) is part of feature A. Features B and C are defined with new boundary contours.

\section{APPLICATION}

\subsection{The multi agent system}

Generalisation constraints and operators were implemented for evaluation in a multi-agent system. Multi-agent systems are, like optimisation methods, constraint-based approaches. Agents can consider different operations and perform several plans to choose the best result. Such model provides more flexibility than a condition-action model where each action is determined by a set of fixed rules Harrie and Weibel (2007). Furthermore, agents can perform both discrete and continuous operations and thus operations on the data structure and the snake model can both be included in the agent model.

A multi-agent system for isobath generalisation was introduced by Zhang and Guilbert (2011). Following the approach developed by Ruas (1999), map objects are defined as two different types of agents: isobaths are micro-agents and features formed by groups of isobaths are meso-agents. Each type of agent has its own constraints, evaluation methods detecting conflicts and operators performing generalisation actions. Zhang and Guilbert (2011) detail a list of constraints and actions for both types of agent and discuss the interest of the approach for feature-driven generalisation but plan evaluation and communication between agents are not addressed.

Each feature agent goes through a series of steps summarised in Figure 6. The feature first evaluates if generalisation must be performed by communicating with the contour agent that defines its boundary. The feature passes information about neighbouring features and admissible direction of deformation. Based on this information, the contour checks if any area or distance conflict occurs and returns the result to the feature.

If some constraint is violated, a list of possible actions is set depending on the feature type. For a peak, possible actions are deformation, enlargement and aggregation. For a pit, possible actions are deletion and deformation. Deformation is applied when the distance constraint is violated. The feature agent first triggers continuous operations. The feature calls again the contour agent which minimises its energy. During the process, the isobath checks if any other conflict occurs. For example, when enlarging a peak, it may move closer to another peak, i.e. to a distance smaller than $\varepsilon_{\text {agr }}$. In that case, the action is rolled back 


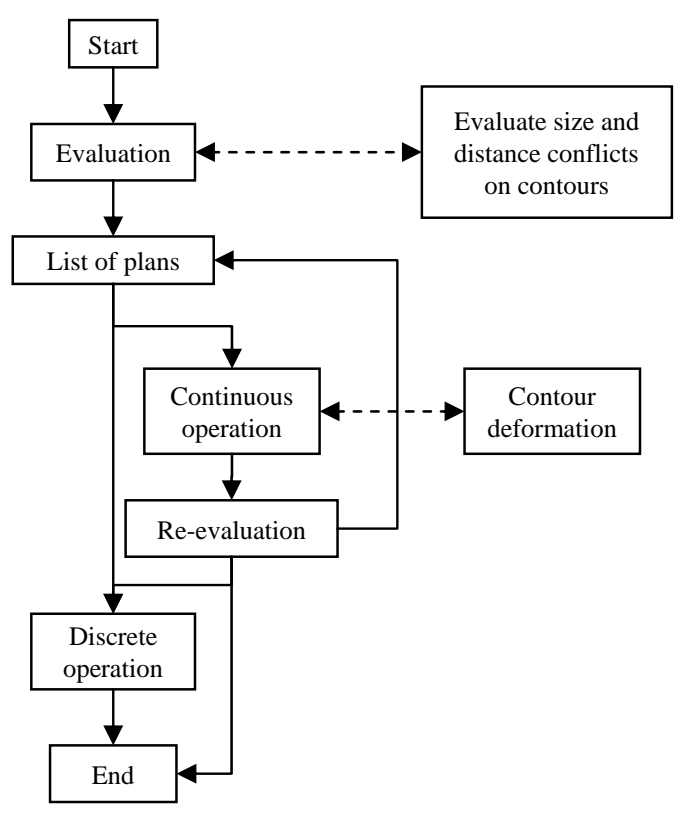

Figure 6: Flowchart of the feature generalisation process.

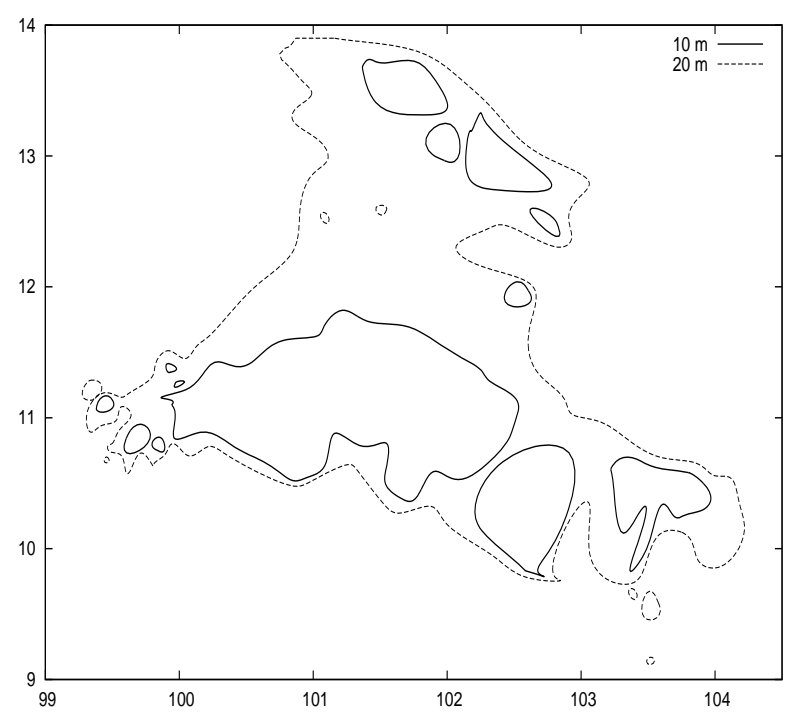

Figure 7: Boundary contours before generalisation. Units in $\mathrm{cm}$

and the next action in the plan, aggregation is performed. Once continuous operation is completed, the data structure is updated if required (Figure 6). During the process, topological and safety constraints are always maintained as any operation that would violates these constraints would be rejected.

\subsection{Results}

The model was run on a set of bathymetric lines provided by the French Hydrographic and Oceanographic Service (SHOM). As soundings were not included in the model, the area constraint is computed using the centre of the isobath for $\Omega$. Only constraints and operations described above were applied. Local constraints related to isobath smoothness and legibility inside a feature were not considered. An example from a 1:50,000 map is presented in Figure 7. Only contours modelling the boundaries of features are taken into account and generalised.

The result of the feature generalisation process is given in Figure 8. Six feature aggregation and two feature enlargement were per-

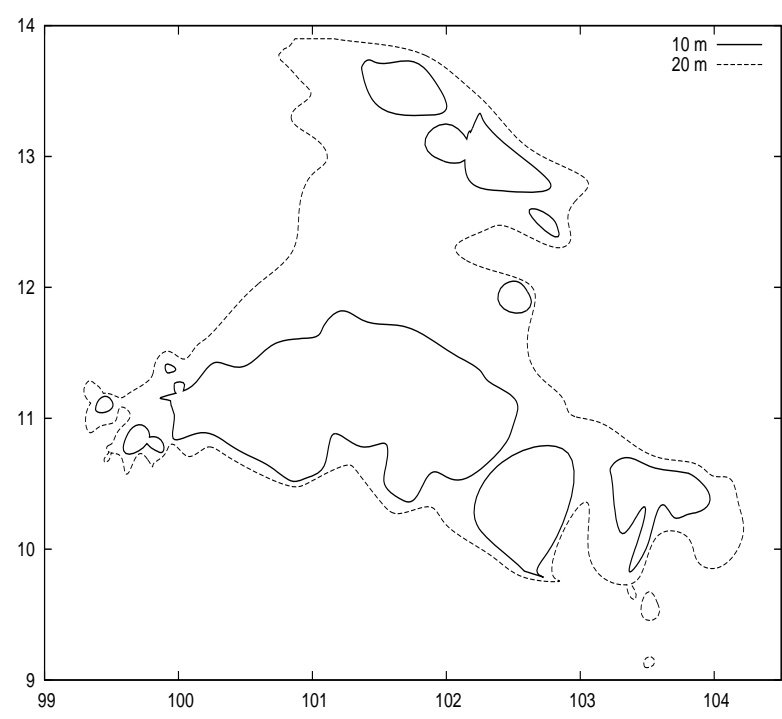

Figure 8: Map after generalisation. Units in $\mathrm{cm}$.

formed on peaks. The process was done automatically and the feature tree was updated preserving the topological information.

Based on the type of feature (peak or pit), agents were able to choose and apply an operator. Plans are indeed limited as a peak is enlarged if there is enough room around otherwise it is aggregated with the closest feature even if several candidates are available. If the aggregated features is large enough, no more aggregation would be done while a human cartographer may have aggregated all neighbouring features. In the bottom part of Figure 8 , a small 20 metre contour was aggregated with the large 20 metre contour which was closer. Shape constraints are not considered as this stage focuses on model generalisation, yielding a merged contour with acute angles. Contours still need to be smoothed in order to fit chart requirements.

\section{CONCLUSION AND FUTURE WORKS}

Although contour generalisation has been addressed in many different ways, whether directly or by generalising the terrains, existing methods do not apply to nautical chart generalisation due to its purpose. Previous works were mostly concerned with providing a legible map and terrain features were not considered in the process while on a chart, contours must be selected and generalised differently according to the type of terrain they represent. Therefore, this paper presents an approach where contours are grouped into features and generalisation is performed on the features. Generalisation constraints and operations are described including continuous transformations (enlargement, aggregation) based on a snake model and updates brought to the data structure.

The operators are introduced in a multi-agent model. Terrain features are processed by generalising their boundary contours according to feature attributes and constraints. In current results, operations are selected and performed automatically. At this stage, constraints on individual contours such as smoothness are not integrated in the model. Micro-agents can evaluate their environment based on information from meso-agents and perform actions in line with (Ruas, 1999) however these actions follow plans set by meso-agents. A next stage is to have contours able to set their own plans to perform operations inside features following Duchêne (2004)'s approach.

In comparison with human generalisation, automatic generalisation tends to preserve more features. A first reason is that the 
method currently does not take into account other elements on the chart. Second is that the method is more conservative because it tends to keep as much information as possible to preserve the morphology while a human cartographer tends to provide a more schematic representation. Soundings were not considered in this example but the constraints and snake energy can be modified to integrate sounding positions in the process. On a longer term, the objective is to integrate soundings as micro-agents and include them in generalisation plans. Sounding selection would be done according to feature attributes and adjacent contours.

\section{ACKNOWLEDGEMENTS}

The work presented in this paper is supported by the Research Grants Council of Hong Kong under General Research Fund grant PolyU 5172/08E. Authors are grateful to the SHOM who provided the bathymetric data.

\section{References}

Ai, T., 2004. A generalization of contour line based on the extraction and analysis of drainage system. In: Proceedings of the XXth ISPRS congress, International Archives of Photogrammetry, Remote Sensing and Spatial Information Sciences, Vol. XXXV, part B4.

Bader, M., 2001. Energy minimization methods for feature displacement in map generalization. $\mathrm{PhD}$ thesis, Universität Zürich.

Bader, M., Barrault, M. and Weibel, R., 2005. Building displacement over a ductile truss. International journal of geographical information science 19(8-9), pp. 915-936.

Beard, K., 1991. Constraints on rule formation. In: B. Buttenfield and R. B. McMaster (eds), Map Generalization, London, pp. 121-135.

Burghardt, D. and Meier, S., 1997. Cartographic displacement using the snakes concept. In: W. Förstner and L. Plümer (eds), Semantic modeling for the acquisition of topographic information from images and maps, Birkhauser Verlag, pp. 59-71.

Chaudhry, O. and Mackaness, W., 2008. Creating mountains out of mole hills: Automatic identification of hills and ranges using morphometric analysis. Transactions in GIS 12(5), pp. 567-589.

Cronin, T., 1995. Automated reasoning with contour maps. Computers and geosciences 21(5), pp. 609-618.

Duchêne, C., 2004. Généralisation cartographique par agents communicants: le modèle CartACom. PhD thesis, Université Paris 6.

Gökgöz, T., 2005. Generalization of contours using deviation angles and error bands. The Cartographic Journal 42(2), pp. 145156.

Guilbert, E. and Saux, E., 2008. Cartographic generalisation of lines based on a B-spline snake model. International Journal of Geographical Information Science 22(8), pp. 847-870.

Harrie, L. and Weibel, R., 2007. Modelling the overall process of generalisation. In: W. Mackaness, A. Ruas and L. Sarjakoski (eds), Generalisation of Geographic Information: Cartographic Modelling and Applications, Elsevier Science, Amsterdam, pp. 67-87.
Kass, M., Witkin, A. and Terzopoulos, D., 1987. Snakes: active contour models. In: Proceedings of International Conference on Computer Vision, IEEE Computer Society Press, Washington DC, pp. 259-268.

Kweon, I. S. and Kanade, T., 1994. Extracting topographic terrain features from elevation maps. CVGIP: Image Understanding 59(2), pp. 171-182.

Li, Z. and Sui, H., 2000. An integrated technique for automated generalization of contour maps. The Cartographic Journal 37(1), pp. 29-37.

Mackaness, W. and Steven, M., 2006. An algorithm for localised contour removal over steep terrain. The Cartographic Journal 43(2), pp. 144-156.

Matuk, K., Gold, C. and Li, Z., 2006. Skeleton based contour line generalization. In: A. Riedl, W. Kainz and G. A. Elmes (eds), Progress in Spatial Data Handling, Springer Berlin Heidelberg, pp. 643-658.

Mustière, S., 1998. GALBE: Adaptive generalisation, the need for an adaptive process for automated generalisation, an example on roads. In: GIS Planet 98, CDROM.

Nickerson, B., 1988. Automated cartographic generalization for linear features. Cartographica 25(3), pp. 15-66.

NOAA, 1997. Nautical Chart User's Manual. National Oceanic and Atmospheric Administration, U.S. Department of Commerce.

Ruas, A., 1999. Modèle de généralisation de données géographiques à base de contraintes et d'autonomie. $\mathrm{PhD}$ thesis, Université de Marne-la-Vallée.

Ruas, A. and Plazanet, C., 1997. Strategies for automated generalization. In: M. Kraak, M. Molenaar and E. Fendel (eds), Advances in GIS research II, Proceedings of the $7^{\text {th }}$ International Symposium on Spatial Data Handling, Taylor \& Francis, London, pp. 319-336.

Wang, Z. and Müller, J.-C., 1998. Line generalization based on analysis of shape characteristics. Cartography and Geographic Information Systems 25(1), pp. 3-15.

Zhang, X. and Guilbert, E., 2011. A multi-agent system approach for feature-driven generalization of isobathymetric line. In: A. Ruas (ed.), Advances in Cartography and GIScience. Selection from ICC 2011, Paris, Vol. 1, Springer, pp. 477-495. 